\title{
Use of ventilator associated pneumonia bundle and statistical process control chart to decrease VAP rate in Syria
}

Reem Alsadat, Hussam Al-Bardan, Mona N. Mazloum¹, Asem A. Shamah², Mohamed F. E. Eltayeb ${ }^{1}$, Ali Marie', Abdulrahman Dakkak ${ }^{3}$, Ola Naes ${ }^{2}$, Faten Esber, Ibrahim Betelmal ${ }^{4}$, Mazen Kherallah ${ }^{5}$

Department of Internal Medicine, Al-Mouassat Hospital, ${ }^{1}$ Critical Care Services, General Assembly of Damascus Hospital, ${ }^{2}$ Al-Bassel Heart Institute, ${ }^{3}$ Critical Care Services, Ibn Al-Nafees Hospital, ${ }^{4}$ World Health Organization (Damascus Office), ${ }^{5}$ Adult Critical Care Medicine, King Faisal Specialist Hospital and Research Center, Riyadh, Saudi Arabia

\begin{tabular}{|c|}
\hline Access this article online \\
\hline Website: www.avicennajmed.com \\
\hline DOI: 10.4103/2231-0770.110736 \\
\hline Quick Response Code: \\
$\square$ \\
\hline
\end{tabular}

\section{ABSTRACT}

OBJECTIVE: Implementation of ventilator associated pneumonia (VAP) bundle as a performance improvement project in the critical care units for all mechanically ventilated patients aiming to decrease the VAP rates. MATERIALS AND METHODS: VAP bundle was implemented in 4 teaching hospitals after educational sessions and compliance rates along with VAP rates were monitored using statistical process control charts. RESULTS: VAP bundle compliance rates were steadily increasing from 33 to $80 \%$ in hospital 1, from 33 to $86 \%$ in hospital 2 and from 83 to $100 \%$ in hospital 3 during the study period. The VAP bundle was not applied in hospital 4 therefore no data was available. A target level of $95 \%$ was reached only in hospital 3 . This correlated with a decrease in VAP rates from 30 to 6.4 per 1000 ventilator days in hospital 1 , from 12 to 4.9 per 1000 ventilator days in hospital 3 , whereas VAP rate failed to decrease in hospital 2 (despite better compliance) and it remained high around 33 per 1000 ventilator days in hospital 4 where VAP bundle was not implemented CONCLUSION: VAP bundle has performed differently in different hospitals in our study. Prevention of VAP requires a multidimensional strategy that includes strict infection control interventions, VAP bundle implementation, process and outcome surveillance and education.

Key words: Bundle, mechanical ventilation, prevention, ventilator associated pneumonia

\section{INTRODUCTION}

Ventilator-associated pneumonia (VAP) is among the most common infections in patients requiring endotracheal tubes with mechanical ventilation. It has been reported to occur in $9 \%$ to $27 \%$ of all intubated patients. ${ }^{[1]}$ VAP is associated with increased hospital costs, ${ }^{[2]}$ a greater number of days in the intensive care unit (ICU), longer duration of mechanical ventilation, and higher mortality. ${ }^{[3-6]}$ The overall rate of ventilator-associated pneumonia (VAP) was 13.6 per 1000 ventilator days according to International Nosocomial Infection Control Consortium (INICC) report data summary for 2003-2008 compared to 3.3 per 1000 ventilator-days in the US National Healthcare Safety
Network (NHSN; formerly the National Nosocomial Infection Surveillance system (NNIS)). ${ }^{[7]}$

Because multiple factors contribute to the high risk of ventilator associated pneumonia, a multi-strategy approach is required to prevent such infections. The Institute of Health Improvement (IHI) has developed a ventilator bundle that incorporates several strategiesto prevent morbidity associated with the ventilator. ${ }^{[8]}$ The IHI ventilator bundle has been broadly adopted by many hospitals as part of the effort to reduce VAP. The use of VAP bundle has been reported to decrease the incidence of VAP in the intensive care units (ICUs) in few studies. ${ }^{[9]}$ 


\section{OBJECTIVES}

The main objective of this study was to implement VAP bundle as a performance improvement project in the critical care units for all mechanically ventilated patients aiming to decrease the VAP rates over the 8 month study period.

\section{MATERIALS AND METHODS}

Patients were recruited between March and November 2011 in 4 major tertiary care teaching hospitals in Damascus: Al-Mouassat Hospital (hospital1), Damascus Hospital (hospital 2), Al-Bassel Heart Institute (hospital 3) and Ibn Alnafees Hospital (hospital4). Patients were included if they were mechanically ventilated for more than $24 \mathrm{~h}$ and were at least $14 \mathrm{yrs}$ of age. A day on mechanical ventilation was defined as any $24 \mathrm{~h}$ period in which the patient required any mode of controlled or assisted ventilation, with the exception of intermittent application of continuous positive airway pressure for atelectasis prophylaxis. Standard unit measures of general critical care were applied to all patients, including the current practice of hand washing, daily oral care, and tracheal suction. The ventilation tubing systems remained unchanged. The medical management, antibiotic therapy, and weaning from the ventilator were left to the treating physician's discretion. Assignment of patients to nursing staff and clinicians was not controlled by the study protocol.

The ventilator bundle was accommodated from the Institute of Health Care Improvement (IHI). ${ }^{[10,11]}$ The bundle elements included elevation of the head of the bed to between 30-45 degrees, daily sedation vacation, daily assessment of readiness to wean, peptic ulcer disease prophylaxis, and deep venous thrombosis prophylaxis if not contraindicated.

Deep Venous Thrombosis (DVT) or stress ulcer prophylaxis was defined as being achieved if the treating physician prescribed the appropriate medication in the medical record and the administration of the medication was signed by the nurse. Complete bundle adherence was defined as fulfillment of all five elements in patients on mechanical ventilation. ${ }^{[12]}$

Ventilator associated pneumonia (VAP) was defined as per Center of Disease Control (CDC) as a pneumonia that occurs in a patient who was intubated and ventilated at the time of or within $48 \mathrm{hrs}$ before the onset of the event. Pneumonia was identified using a combination of radiological, clinical, and laboratory criteria. ${ }^{[13]}$ VAP was clinically diagnosed based on progressive infiltrate in chest $\mathrm{X}$-rays, leukocytosis, pyrexia above $38.5^{\circ} \mathrm{C}$, purulent secretions, and crepitations in lungs. VAP rates were calculated based on occurrences per 1000 ventilator days and monitored on a monthly basis throughout the project period.

Interventions included an initial educational workshop for the stakeholders from different participating hospitals with the emphasis on standards of practice, nursing support and physician leadership, participation, and collaboration. A multidisciplinary team was formed at every hospital and included 1-2 physicians and 1-2 nurses with a team leader who was selected based on experience, knowledge and commitment. Staff education with bedside mentoring and skills competency documentation was done for all the nurses and physicians in the specific unit. Teams conducted regular daily rounds on all ventilated patients and recorded compliance with the five elements of the VAP bundle. Leaders were instructed to set up regularly scheduled meetings with teams to hear about progress, barriers, and next steps.

Data was collected daily and plotted on the Statistical Control Chart (SPC) on a weekly basis, then analyzed at the project manager level at the end of each month and a progress report was generated that included up to date results and recommendations. These reports were communicated directly to the members of the team and charts were posted in the specific unit. Regular monthly follow-up meetings and frequent staff education sessions in individual units to ensure the quality of the implementation process were enforced based on the progress report and the feedback from different units.

Statistical process control chart was used to monitor the process of compliance with the individual bundle elements as well as the whole bundle. ${ }^{[14]}$ Process stability is defined as the ability of the process to perform in a predictable manner over time without exhibiting any of the special cause variations and process capability is defined as a process that is able to produce results that meet the desired goal. The target was set in our study as a special cause variation with at least 6 data points in a row showing a stepwise increase causing an improvement in the VAP bundle compliance rate to above $95 \%$. The process was then considered stable if no more special cause variations observed over time and capable when the compliance rate remained above $95 \%$.

\section{RESULTS}

VAP bundle compliance rate was steadily increasing from 33 to $91 \%$ during the initial implementation period in hospital 1 before it started to decline to $64.1 \%$, necessitating more efforts to reinforce the implementation of the bundle elements that resulted in an increase in the compliance 
rate to around $80 \%$. This correlated with a decrease in the VAP rate from 30 per 1000 ventilator days down to 6.4 per 1000 ventilator days [Figure 1]. In hospital 2, the VAP bundle compliance rate has continued to increase steadily from 33 to $86 \%$ whereas the VAP rate fluctuated in the range of 13-24 per 1000 ventilator days [Figure 2]. In hospital 3, the VAP bundle compliance rate was $83 \%$ at the start of the study and increased to $100 \%$ during the last 2 months of the study with a decrease in the VAP rate from 12 to 4.9 per 1000 ventilator days [Figure 3]. The VAP bundle was not applied in hospital 4 due to different reasons related to resource availability; therefore no data was available but they reported VAP rates for part of the study period (May-August) with very high VAP rates during May and June and rates of 33 and 34 per 1000 ventilator days during the months of July and August respectively [Figure 4].

Statistical process control chart that monitored the implementation of VAP bundle on a weekly basis has revealed a special cause variation with six or more steadily increasing data points that reflected an improvement in compliance rate in hospital 1,2 and 3. The target level of $95 \%$ compliance rate was only achieved in hospital 3 .

\section{DISCUSSION}

The IHI has developed a ventilator bundle that incorporates several strategies to prevent morbidity associated with the ventilator. Three elements of this bundle target VAP while 2 elements address prevention of stress ulcers and

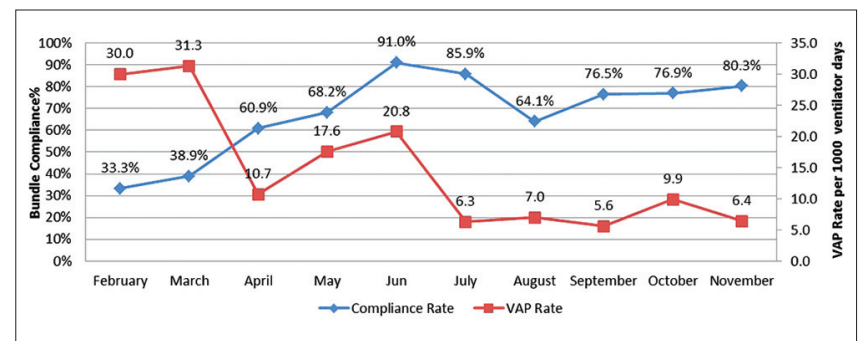

Figure 1: VAP bundle compliance rates and VAP rates in hospital (1) over the study period

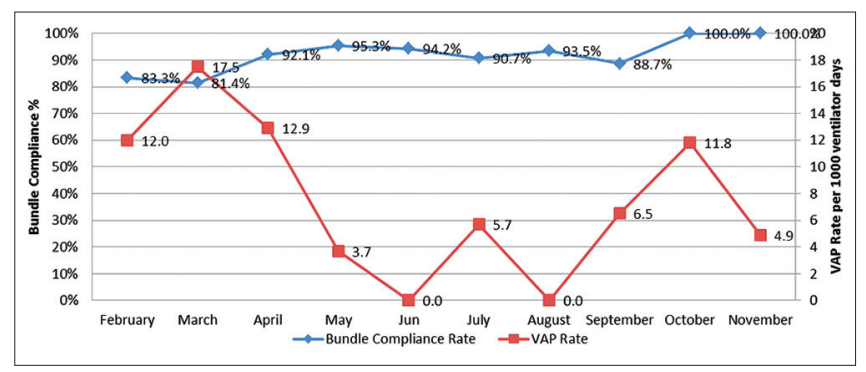

Figure 3: VAP bundle compliance rates and VAP rates in hospital (3) over the study period thromboembolic disease ${ }^{[8]}$ The IHI ventilator bundle has been broadly adopted by many hospitals as part of an effort to reduce VAP. Several studies have showed the benefit of the individual elements of the VAP bundle in preventing VAP. ${ }^{[9]}$ In 2 small studies, aspiration and VAP were reduced almost 3-fold for patients with the head of the bed at $45^{\circ}$ compared with patients who were supine. ${ }^{[15,16]}$ Daily interruption of sedation has been included in the bundle as it shows that it shortens the duration of mechanical ventilation. ${ }^{[17]}$ Spontaneous awakening trials added to spontaneous breathing trials are associated with more rapid ventilator weaning, fewer ICU days, and shorter hospitalizations. ${ }^{[18]}$ Despite broad implementation of a bundled strategy aimed at preventing ventilator-associated adverse events in many hospitals, the ability of the bundle to prevent VAP has not been definitively established with high-quality studies. Two studies that have reported on the effectiveness of implementing the 3 components of the bundle intended to prevent VAP did not report adherence rates to the bundle. ${ }^{[19,20]}$ Another study evaluating these components of the bundle reported a 95\% adherence with the bundle and an associated reduction in VAP, but investigators acknowledged that the reduction may have been related to a concurrent improvement program that focused on care of the ventilated patient with multidisciplinary teams and daily goal setting rather than bundle adherence. ${ }^{[11]}$ No large randomized study has demonstrated that reducing VAP using any VAP prevention strategy, including those in the IHI bundle, is associated with improvements in clinical outcomes. More recently a multidimensional strategy that included a bundle of infection control interventions,

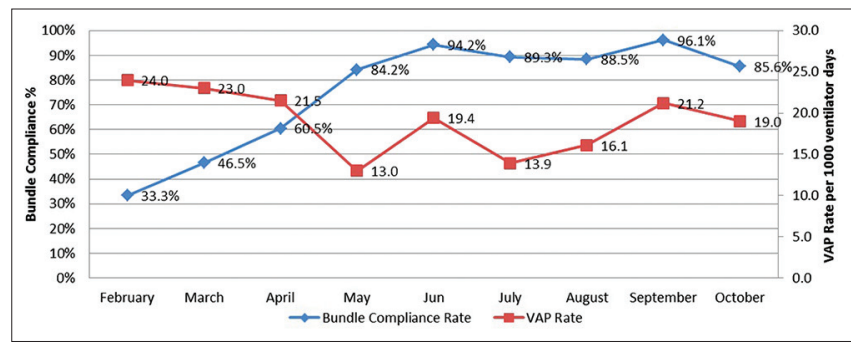

Figure 2: VAP bundle compliance rates and VAP rates in hospital (2) over the study period

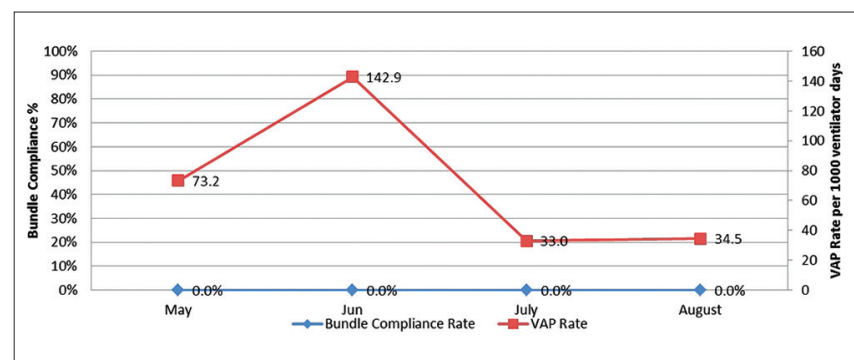

Figure 4: VAP bundle compliance rates and VAP rates in hospital (4) over the study period 
education, outcome surveillance, process surveillance, feedback on VAP rates, and performance feedback on infection control practices associated with a significant reduction in VAP rate in NICUs in developing countries. ${ }^{[21]}$

Our study demonstrates that if no VAP bundle is implemented, it is obvious that VAP rates will continue to remain high as is the case with hospital 4 . On the other hand, even though there was an improvement in the process of bundle compliance rate in hospital 1 and 2 with special cause variation and the process became stable but the target compliance rate of $95 \%$ was never reached. Therefore, the process would have been considered stable but not capable in both hospitals compared to the improved process in hospital 3 to the target level of $95 \%$ and maintained above that level where the process would be considered stable and capable. This process capability in hospital 3 was reflected in a remarkable decrease in the VAP rate whereas in hospital 2 where the process is not capable; there was no notable decrease in VAP rate. In hospital 1 on the contrary; where the bundle implementation process has improved and became stable but not capable; the VAP rate showed a remarkable improvement over the course of the study. Other infection control practices and measures may have played a role in the difference in performance of VAP bundle among the study hospitals. However, the study did not look into the different infection control measures at the study hospitals- an intervention that may have added more value to our study. The results of this study should be looked at in the context of the major challenges that face the implementation of quality improvement project in a resource limited environment, including lack of nursing leadership, individual variability, high turnover of nurses, lack of structured competency skills testing, lack of overall safety culture, communication and teamwork in ICU, non-familiarity with quality projects, lack of policies and procedures, poor documentation and communication, and validity of data collection. Nevertheless this study demonstrates that with dedication and commitment, a tangible improvement can be made despite all challenges. Critical factors that should be taken into consideration for the success of this type of quality project include creating organizational alignment by declaring eliminating hospital acquired infections as an organizational breakthrough goal, having the organization's executive leadership highly engaged in the project, coordination by an experienced and effective project leader and manager, collaboration by multidisciplinary project teams, and promoting transparency of results across the organization.

\section{CONCLUSION}

VAP bundle that is widely implemented as an effective method to reduce VAP rates has performed differently in different hospitals in our study. Prevention of VAP requires concerted efforts on the part of hospital administration, physicians, and ICU personnel. The program must be evidence-based, maintained, and accepted by ICU personnel. Monitoring and collection of data should be strict, objective, and transparent. A multidimensional strategy that includes strict infection control interventions, VAP bundle implementation, process and outcome surveillance and education is needed to reduce VAP rates.

\section{REFERENCES}

1. American Thoracic Society; Infectious Diseases Society of America. Guidelines for the management of adults with hospital-acquired, ventilator-associated, and healthcare-associated pneumonia. Am J Respir Crit Care Med 2005;171:388-416.

2. Warren DK, Shukla SJ, Olsen MA, Kollef MH, Hollenbeak CS, Cox MJ, et al. Outcome and attributable cost of ventilator-associated pneumonia among intensive care unit patients in a suburban medical center. Crit Care Med 2003;31:1312-7.

3. Bekaert M, Timsit JF, Vansteelandt S, Depuydt P, Vésin A, Garrouste-Orgeas M, et al. Attributable mortality of ventilator- associated pneumonia: A reappraisal using causal analysis. Am J Respir Crit Care Med 2011;184:1133-9.

4. Heyland DK, Cook DJ, Griffith L, Keenan SP, Brun-Buisson C. The attributable morbidity and mortality of ventilator-associated pneumonia in the critically ill patient. Am J Respir Crit Care Med 1999;159:1249-56.

5. Craven D. Epidemiology of ventilator-associated pneumonia. Chest 2000;117:186S-7S.

6. Rello J, Ollendorf D, Oster G, Vera-Llonch M, Bellm L, Redman R, et al. Epidemiology and outcomes of ventilator-associated pneumonia in a large US database. Chest 2002;122:2115-21.

7. Rosenthal VD, Maki DG, Jamulitrat S, Medeiros EA, Todi SK, Gomez DY, et al. International Nosocomial Infection Control Consortium (INICC) report, data summary for 2003-2008, issued June 2009. Am J Infect Control 2010;38:95-104.

8. Implement the IHI ventilator bundle. Institute for Healthcare Improvement. Available from: http://www.ihi.org/knowledge/Pages/ Changes/ImplementtheVentilatorBundle.aspx. (Last accessed on 2012 Jan 24).

9. Cocanour CS, Peninger M, Domonoske BD, Li T, Wright B, Valdivia A, et al. Decreasing ventilator-associated pneumonia in a trauma ICU. J Trauma 2006;61:122-9.

10. Youngquist P, Carroll M, Farber M, Macy D, Madrid P, Ronning J, et al. Implementing a ventilator bundle in a community hospital. Jt Comm J Qual Patient Saf 2007;33:219-25.

11. Resar R, Pronovost P, Haraden C, Simmonds T, Rainey T, Nolan T. Using a bundle approach to improve ventilator care processes and reduce ventilator associated pneumonia. Jt Comm J Qual Patient Saf 2005;31:243-8.

12. Nolan T, Berwick DM. All-or-none measurement raises the bar on performance. JAMA 2006;295:1168-70.

13. Horan TC, Andrus M, Dudreck MA. CDC/NHSN surveillance definition of health-care definition of health care-associated infection and criteria for specific types of infections in the acute care setting. Am J Infect Control 2008;36:309-32.

14. Johan T, Lundberg J, Ask J, Olsson J, Carli C, Härenstam KP, et al. Application of statistical process control in healthcare improvement: Systematic review. Qual Saf Health Care 2007;16:387-99.

15. Kollef $\mathrm{MH}$. Ventilator-associated pneumonia: A multivariate analysis. JAMA 1993;270:1965-70. 
16. Drakulovic MB, Torres A, Bauer TT, Nicolas JM, Nogué S, Ferrer M. Supine body position as a risk factor for nosocomial pneumonia in mechanically ventilated patients: A randomized trial. Lancet 1999;354:1851-8.

17. Kress JP, Pohlman AS, O'Connor MF, Hall JB. Daily interruption of sedative infusions in critically ill patients undergoing mechanical ventilation. N Engl J Med 2000;342:1471-7.

18. Girard TD, Kress JP, Fuchs BD, Thomason JW, Schweickert WD, Pun BT, et al. Efficacy and safety of a paired sedation and ventilator weaning protocol for mechanically ventilated patients in intensive care (Awakening and Breathing Controlled trial): A randomized controlled trial. Lancet 2008;371:126-34.

19. Berriel-Cass D, Adkins FW, Jones P, Fakih MG. Eliminating nosocomial infections at Ascension Health. Jt Comm J Qual Patient Saf 2006;32:612-20.

20. Unahalekhaka A, Jamulitrat S, Chongsuvivatwong V, Øvretveit J. Using a collaborative to reduce ventilator-associated pneumonia in Thailand. Jt Comm J Qual Patient Saf 2007;33:387-94.

21. Rosenthal VD, Rodríguez-Calderón ME, Rodríguez-Ferrer M, Singhal T, Pawar M, Sobreyra-Oropeza M, et al. Findings of the International Nosocomial Infection Control Consortium (INICC), Part II: Impact of a multidimensional strategy to reduce ventilator-associated pneumonia in neonatal intensive care units in 10 developing countries. Infect Control Hosp Epidemiol 2012;33:704-10.

Cite this article as: Alsadat $\mathrm{R}, \mathrm{Al}$-Bardan $\mathrm{H}$, Mazloum MN, Shamah $\mathrm{AA}$, Eltayeb MF, Marie A, Dakkak A, Naes O, Esber F, Betelmal I, Kherallah M. Use of ventilator associated pneumonia bundle and statistical process control chart to decrease VAP rate in Syria. Avicenna J Med 2012;2:79-83.

Source of Support: Nil, Conflict of Interest: None declared.

\section{“QUICK RESPONSE CODE” LINK FOR FULL TEXT ARTICLES}

The journal issue has a unique new feature for reaching to the journal's website without typing a single letter. Each article on its first page has a "Quick Response Code". Using any mobile or other hand-held device with camera and GPRS/other internet source, one can reach to the full text of that particular article on the journal's website. Start a QR-code reading software (see list of free applications from http://tinyurl.com/yzlh2tc) and point the camera to the QR-code printed in the journal. It will automatically take you to the HTML full text of that article. One can also use a desktop or laptop with web camera for similar functionality. See http://tinyurl.com/2bw7fn3 or http://tinyurl.com/3ysr3me for the free applications. 